\title{
Traffic Modeling and State Feedback Control for Metro Lines
}

\author{
V: Van Breusegem, G. Campion, and G. Bastin
}

\begin{abstract}
This paper deals with traffic modeling and control design for high-frequency metro lines. A complete discrete-event traffic model pointing out the natural instability of metro lines is presented. The traffic stability properties are analyzed and easyto-implement state feedback traffic control algorithms are designed, which guarantee the system stability. Simulations illustrate the methodology.
\end{abstract}

\section{INTRODUCTION}

$\mathrm{H}$ IGH-FREQUENCY metro lines are well known to be naturally unstable. This means that any deviation with respect to the nominal schedule of a given train is amplified with time and disturbs the operation of the other trains. The phenomenon is explained as follows. On a high-frequency line, the passengers arrive randomly at the stations. Hence, the number of passengers waiting at a platform to get on the next train increases with the time elapsed since the departure of the preceding train. If a train is delayed, this time interval, and therefore the number of passengers, become greater than nominally expected. The staying time of the train at the platform depends on the number of passengers exchanged between platform and train and naturally increases too. Hence, the delay of the train is increased from one platform to the next. Conversely, if the next train operation is not delayed, this train will be ahead of schedule since the time interval will be shorter than expected due to the number of passengers at a platform and the corresponding train staying times being less than their nominal values. The same argument shows that the next train is delayed ... and so on.

Traffic control is therefore necessary in order to prevent such instabilities. A minimal traffic control is always implemented on metro lines, by use of traffic lights and other protection devices in order to ensure, according to the security rules, a minimal distance between successive trains and to avoid collisions. The resulting traffic conditions are, however, far from being ideal: the time deviations with respect to the nominal time schedule are large, the distribution of passengers among the trains is excessively nonuniform and the commercial speed is reduced. Thus, more efficient traffic control strategies are therefore necessary, both from a passenger and company viewpoint.

Manuscript received August 28, 1989; revised August 10, 1990 and December 13, 1990. Paper recommended by Associate Editor at Large, M. P. Polis. The work of G. Campion was supported by the F.N.R.S.

The authors are with the Laboratoire d'Automatique, Dynamique et Analyse des Systèmes, Université Catholique de Louvain, Bâtiment Maxwell, Place du Levant 3, B-1348, Louvain-la-Neuve, Belgium.

IEEE Log Number 9100497.
Currently, on most commercial lines, traffic control consists only of resetting the time deviations to zero at selected platforms (generally those of the end of each line) by use of time margins. This solution however requires operating more trains than necessary and consequently, at each instant, several trains are in standby. In this paper, we shall show how efficient control algorithms can be designed which ensure the stability of the operation, do not need time margins and therefore allow operation with a minimal number of trains.

Control algorithms for a string of moving vehicles have been proposed, by Levine and Athans [1] and others [2]-[5]. The approach in [1] is based on the optimal control theory, but it is inconvenient for traffic control of metro lines; the vehicle stopping at fixed points (platforms) is not taken into account. Moreover, the control algorithms are elaborated from mathematical models which are deduced from the motion equations. Therefore, the position, velocity, and acceleration of each vehicle have to be evaluated at each instant.

A more suitable class of mathematical models is obtained from a discrete-event approach. Such models account only for discrete events occurring on the line, e.g., arrival or departure of trains at or from platforms. The corresponding variables are related to both trains and platforms. This approach which is used in this paper, has already been used in order to design optimal-time schedules by means of optimization techniques [6] or to examine the traffic dynamics of an automated transit system [7].

The purpose of this paper is to present a complete discrete-event traffic model pointing out the natural instability of metro lines, to analyze the traffic stability properties, and to design easy-to-implement traffic control algorithms which guarantee the system stability.

The paper is organized as follows. The description of the traffic on sequential metro lines is given in Section II. Two different kinds of lines are considered: open and loop lines. In Section III, the basic linear model of the traffic obtained from a discrete-event approach is presented. This model describes the transfer of a train between two successive platforms. The traffic on open lines is analyzed in Section IV. The concepts of nominal time schedule and time deviations are introduced and a global state-space description of the traffic on open lines is presented. This state-space approach is used to analyze the natural instability of the line and to design traffic control algorithms using state feedback. The stability properties of these algorithms are demonstrated. Section $\mathrm{V}$ is devoted to analyzing the traffic on loop lines. The concept of natural interval which is induced by the cyclic structure of the line is introduced and a global state-space representation 


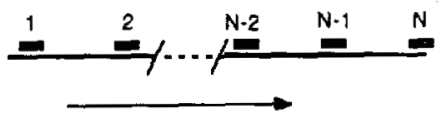

Fig. 1. Example of open line.

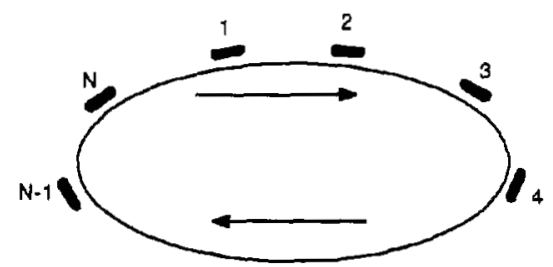

Fig. 2. A loop line with $N$ platforms.

accounting for this structure is deduced from the linear traffic description. Instability properties and traffic control algorithms are presented. Traffic description, when there is no reference time schedule, is then presented and traffic control algorithms valid for this situation are designed. An estimation technique which allows the model to cope with inaccurate prior knowledge of the natural interval is also proposed. Simulation results are given in Section VI to illustrate the methodology.

\section{TRAFFIC Description}

Consider an ordered set of trains running on a metro line constituted by a sequence of platforms where each train has to stop in order to allow passengers to get on and off. Each train and each platform are characterized, respectively, by a number referred to as the train index and the platform index. In this paper, we restrict ourselves to sequential line structures and operating conditions and therefore exclude branching and tree structure lines. This means that:

1) the sequence of platforms encountered by a given train is ordered and the same for all trains on the line;

2) at each platform, the sequence of trains is ordered and the same for all platforms.

The class of sequential lines can be divided into two subclasses: open lines and loop lines which are defined as follows.

Open Lines: An open line is defined as a sequence of $N$ platforms where $M$ trains are operated. The platform indexes and the train indexes vary, respectively, from $\langle 1\rangle$ to $\langle N\rangle$ and from $(1\rangle$ to $\langle M\rangle$. The trains are injected at the first platform $(\langle 1\rangle)$ independently of the past traffic evolution, and leave the line after platform $\langle N\rangle$ (see Fig. 1).

Loop Lines: A loop line is defined as a closed line with $N$ platforms (indexes $\langle 1\rangle$ to $\langle N\rangle$ ) where platform $\langle N\rangle$ is connected to platform $\langle 1\rangle$, and where a given set of trains (indexes $\langle 1\rangle$ to $\langle M\rangle$ ) is periodically operated (see Fig. 2).

For each platform, the ordered sequence of crossing trains

$$
\{1,2, \cdots M, 1,2, \cdots M, 1,2 \cdots,\}
$$

and the ordered sequence of platforms crossed by a given train is

$$
\{1,2, \cdots, N, 1,2, \cdots, N, 1, \cdots,\} .
$$

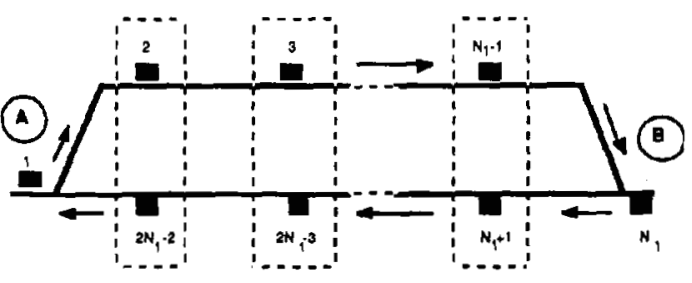

Fig. 3. Example of loop line configuration.

Due to the closed structure of the line, the variables relative to a given train at platform $\langle 1\rangle$ depend on the past traffic evolution and namely on the variables relative to the preceding platform $\langle N\rangle$.

This difference between open and loop lines motivates the two different global traffic descriptions presented in Sections IV and V. However, the analysis of the traffic on open metro lines which will be performed in Section IV has to be seen as an introductive ideal study because most commercial lines are actually operated as loop lines. Consider for instance the classical configuration of a 2-track line between two terminus stations $A$ and $B$ (one track in each direction) (Fig. 3). On course $A B$ the platform numbers are $\langle 1\rangle,\langle 2\rangle, \cdots,\left\langle N_{1}\right\rangle$ and on course BA $\left\langle N_{1}\right\rangle,\left\langle N_{1}+1\right\rangle, \cdots\left(2 N_{1}-2\right\rangle,\langle 1\rangle$. The $\left(N_{1}-2\right)$ couples of platforms $\left\{\left(\langle 2\rangle,\left\langle 2 N_{1}-2\right\rangle\right), \cdots,\left\langle\left\langle N_{1}-1\right\rangle,\left\langle N_{1}\right.\right.\right.$ $+1\rangle)\}$ constitute $\left(N_{1}-2\right)$ physical stations (2 platforms) while $\mathbf{A}$ and $\mathbf{B}$ are two terminus stations with track crossing sections. If a sequence of $M$ trains is operated periodically on the circuit ABA, this line can be considered as a loop line with $\left(2 N_{1}-2\right)$ platforms.

Notation: Throughout this paper we use a two-indexes notation to identify the variables relative to a given train at a given platform: the upper index refers to the train number and the lower index to the platform number. For example $t_{k}^{i}$ will denote the departure instant of the $i$ th train from the $k$ th platform. These indexes however have to be interpreted, respectively, modulo $M$ and modulo $N$. With this convention the indexes of two successive trains can be denoted, without loss of generality, $\langle i\rangle$ and $\langle i+1\rangle$, with $(1 \leq i \leq M)$, (the train $\langle M\rangle$ is followed by train $\langle 1\rangle$ ), and two successive platforms by the indexes $\langle k\rangle$ and $\langle k+1\rangle$, with $(1 \leq k \leq N)$, (platform $\langle N\rangle$ is followed by platform $\langle 1\rangle$ ).

\section{Traffic Modeling}

In this section, we derive the mathematical model relating the departure instants of the different trains from the different platforms.

According to the aforementioned notation, let us denote $t_{k}^{i}$ as the departure instant of train $\langle i\rangle$ from platform $\langle k\rangle$. Obviously, the departure instants of train $\langle i\rangle$ from two successive platforms $\langle k\rangle$ and $\langle k+1\rangle$ are related by

$$
t_{k+1}^{i}=t_{k}^{i}+r_{k}^{i}+s_{k+1}^{i}
$$

where $r_{k}^{i}$ is the running time of train $\langle i\rangle$ from $\langle k\rangle$ to $\langle k+1\rangle$ and $s_{k}^{i}$ is the staying time of train $\langle i\rangle$ at platform $\langle k\rangle$.

In order to further model $r_{k}^{i}$ and $s_{k}^{i}$, we introduce four basic assumptions. 


\section{Basic Assumptions}

A1: The operating conditions (number of trains, desired interval between successive trains, number of passengers arriving at a given platform per second, ...) are constant.

A2: The running time of a train between two successive platforms $\left(r_{k}^{i}\right)$ does not depend on the number of passengers on the train.

A3: The staying time of a train at a platform depends linearly on the number of passengers getting on the train.

A4: The number of passengers to be embarked on train $\langle i\rangle$ at platform $\langle k\rangle$ is proportional to the interval between the departure instants of the successive trains $\langle i-1\rangle$ and $\langle i\rangle$ from platform $\langle k\rangle$.

Assumption Al is introduced only in order to simplify the analysis and can easily be relaxed. The three assumptions A2, A3, A4 can be replaced by more sophisticated modeling assumptions. It is possible, for instance, to take into account the load (i.e., the numbers of passengers) on the trains and to relate the staying time not only to the number of embarking passengers (like in A3) but also to the number of passengers getting off the train (see [8], [9]). Simulation results obtained with such more sophisticated models are, however, not significantly different, as far as traffic analysis is concerned.

From $\mathrm{A} 1$ and $\mathrm{A} 2$, the running time can be expressed as follows

$$
r_{k}^{i}=R_{k}+u_{k}^{i}+w 1_{k}^{i}
$$

where $R_{k}$ is the nominal running time from $\langle k\rangle$ to $\langle k+1\rangle$, $u_{k}^{i}$ is the control action applied to train $\langle i\rangle$ between $\langle k\rangle$ and $\langle k+1\rangle$ in order to increase $\left(u_{k}^{\mathrm{i}} \geq 0\right)$ or to decrease $\left(u_{k}^{i}<0\right)$ the running time, and $w 1_{k}^{i}$ is a disturbance term.

From $\mathrm{A} 1, \mathrm{A3}, \mathrm{A4}$, the staying time can be modeled as

$$
s_{k+1}^{i}=S+c_{k+1}\left(t_{k+1}^{i}-t_{k+1}^{i-1}\right)+w 2_{k+1}^{i}
$$

where $S$ is the minimal staying time at a platform, when no passenger gets on the train and the doors are closed as soon as possible, $c_{k+1}$ is the delay rate representing the effect of the time interval between the departure instants of two successive trains (A4), and $w 2_{k+1}^{i}$ is a disturbance term.

In relations (2) and (3), $S, R_{k}$, and $c_{k}$ are parameters to be estimated. $S$ and $R_{k}$ can be evaluated from the operating conditions while the $c_{k}$ have to be estimated by linear regression on a large number of observations $\left(t_{k}^{i}, s_{k}^{l}\right)$ at each platform, according to (3). Usual values of $c_{k}$ are in the range 0.01 to 0.05 (see [8] for examples relative to Brussels metro lines).

Using (2) and (3), the relation (1) can be rewritten as

$$
\left(1-c_{k+1}\right) t_{k+1}^{i}=t_{k}^{i}-c_{k+1} t_{k+1}^{i-1}+S+R_{k}+u_{k}^{i}+w_{k}^{i}
$$

where $w_{k}^{i}=w 1_{k}^{i}+w 2_{k+1}^{i}$.

This relation between the departure instants of the trains is used throughout the paper. It must be pointed out that the admissible control actions and disturbances are bounded in order to always satisfy the security requirements which are in place to prevent collisions between trains.

Equation (4) gives a local description of the traffic behav- ior relating to two successive trains and two successive platforms. The complete set of departure times $t_{k}^{i}(i=$ $1, \cdots, M ; k=1, \cdots, N)$ corresponds to a global description of the traffic.

\section{Traffic Analysis for Open Lines}

To begin the analysis, we consider the simple case of an open line, constinted by a sequence of $N$ platforms (see Fig. 1). We first introduce in Section IV-A the concept of a nominal schedule and we describe the traffic dynamics in terms of the time deviations between the actual system behavior and this nominal schedule. The intrinsic instability of the system is then analyzed in Section IV-B. Finally, stabilization of the traffic dynamics by state feedback is discussed in Section IV-C.

\section{A. Traffic Description with Reference to a Nominal Schedule}

Nominal Schedule: We assume that an ideal traffic plan has been established for the line under consideration. It takes the form of a nominal time schedule which is defined as the set of the nominal departure instants $T_{k}^{i}$ for each train at each platform on the line. This nominal time schedule is characterized by a constant time interval $H$ between successive trains, i.e.,

$$
H=T_{k}^{i+1}-T_{k}^{i} .
$$

It must be coherent with the natural dynamics of the line, i.e., it must satisfy the basic relation (4) in the absence of control and disturbances $\left(u_{k}^{i}=w_{k}^{i}=0\right)$.

$$
T_{k+1}^{i}=T_{k}^{i}+c_{k+1} H+S+R_{k}
$$

Initial Conditions: For consistency in our subsequent developments, some care must be taken with the initial conditions of (4). We need to introduce a "fictitious initial train" and a "fictitious initial platform." The fictitious initial train, with index $\langle i\rangle=0$, is supposed to be "exactly on time" at each station, i.e., $t_{k}^{0}=T_{k}^{0}, \forall k$. The fictitious initial platform, with index $\langle k\rangle=0$, is such that all the trains are supposed to be exactly "on time" at this platform, i.e., $t_{0}^{i}=T_{0}^{i}, \forall i$.

Time Deviations: Define $x_{k}^{i}$ as the deviation of the actual departure instant $t_{k}^{i}$ from its nominal value $T_{k}^{i}$, i.e.,

$$
x_{k}^{i} \triangleq t_{k}^{i}-T_{k}^{i} \text {. }
$$

Then, the basic dynamical equation (4) is rewritten as follows:

$\left(1-c_{k+1}\right) x_{k+1}^{i}+c_{k+1} x_{k+1}^{i-1}=x_{k}^{i}+u_{k}^{i}+w_{k}^{i}$,

$$
k \geq 0, i \geq 1 \text {. }
$$

For given sequences of control actions and disturbances $\left(u_{k}^{i}\right.$ and $w_{k}^{i}$ ), (7) describes completely the evolution of the set of time deviations $\left\{x_{k}^{i}\right\}$.

\section{B. Intrinsic Instability of the Traffic Behavior}

We now use (7) to emphasize the natural instability of a metro line. Define the $I$-dimensional vector $X_{k}^{S}$ of the time 
deviations of the first $I$ trains on the line, at a given platform $\langle k\rangle$

$$
X_{k}^{S} \triangleq\left[x_{k}^{1} \cdots x_{k}^{I}\right]^{T}
$$

This vector will serve as the state vector of a state-space representation (8) which will be referred to as the "station sequential model" (see [8]). From (7), it is easily shown that, without control or disturbances $\left(u_{k}^{i}=w_{k}^{i}=0\right.$ ), the evolution of $X_{k}^{S}$ satisfies the following linear equation:

$$
X_{k+1}^{s}=A_{k}^{s} X_{k}^{S}
$$

where

$$
A_{k}^{S}=\left[\begin{array}{cccc}
1-c_{k+1} & & & 0 \\
c_{k+1} & 1-c_{k+1} & \\
0 & \ddots & \ddots & \\
& & c_{k+1} & 1-c_{k+1}
\end{array}\right]^{-1}
$$

$A_{k}^{S}$ is lower triangular with diagonal elements $1 /\left(1-c_{k+1}\right)$ $>1$. As the system (8) is not stationary, we cannot immediately conclude concerning its stability. Define the mean-square value of the time deviations of the $I$ trains at a given platform $\langle k\rangle$

$$
\Gamma_{k}=\frac{1}{I} \sum_{i=1}^{I}\left(x_{k}^{i}\right)^{2}
$$

This index reflects the quality of the line operation: if all trains are running under nominal operation mode, this index is equal to zero. With this definition and using the state-space representation (8), we have the following instability result.

Theorem 1: For $c_{k}>0$, if the mean-square value of the time deviations at a given platform is greater than zero, then this mean-square value increases from platform to platform, i.e.,

$$
\Gamma_{k+1}>\Gamma_{k}, \quad k \geq 1 .
$$

Proof: See the Appendix, A.1.

This theorem clearly shows that a metro line is intrinsically an unstable system, whatever the traffic dynamics (i.e., whatever the positive values of the $c_{k}$ 's): any initial time deviation at the first platform is propagated and exponentially amplified along the line.

\section{Traffic Control Using State Feedback}

In this section, we shall present a feedback control law which guarantees system stabilization. The control law will be designed by minimizing a performance index which reflects the operational objectives. Since the traffic description equation (7) is linear with respect to the time deviations, we choose a quadratic performance index in order to reduce the problem to a standard LQ problem resulting in a linear state feedback control. It can be seen, however, that the state-space representation (8) is not suited for an on-line state feedback control implementation: since the state vector components $\left(x_{k}^{i}, i=1, \cdots, I\right)$ are relative to the $I$ trains, the control to be applied to train $\langle i\rangle$ at platform $\langle k\rangle$ would be a function of the $l$ corresponding time deviations including the future (and therefore unknown) time deviations. Before formulating a convenient alternative state model, we introduce the following matrix notation.

Matrix Notation: $D_{1}\left(N ; a_{1}, a_{2}, \cdots, a_{N}\right)$ denotes a diagonal matrix of dimension $N$ parametrized by the successive values of the diagonal components $a_{1}, \cdots, a_{N}$

$$
D_{1}\left(N ; a_{1}, a_{2}, \cdots, a_{N}\right)=\left[\begin{array}{llll}
a_{1} & & & \\
& a_{2} & 0 & \\
& & \ddots & \\
0 & & & a_{N}
\end{array}\right]
$$

$D_{2}\left(N ; a_{1}, a_{2}, \cdots, a_{N} ; b_{1}, \cdots, b_{N-1}\right)$ is a square matrix of dimension $N$ of the following form:

$$
\begin{aligned}
D_{2}\left(N ; a_{1}, a_{2}, \cdots, a_{N} ; b_{1}, b_{2}, \cdots, b_{N-1}\right) & \\
& \equiv\left[\begin{array}{cccc}
a_{1} & & \\
b_{1} & a_{2} & 0 & \\
& \ddots & \ddots & \\
0 & & b_{N-1} & a_{N}
\end{array}\right]
\end{aligned}
$$

parametrized by the successive values of the diagonal components $a_{1}$ to $a_{N}$ and by the successive values of the subdiagonal components $b_{1}$ to $b_{N-1}$.

State-Space Formulation: We first introduce the following definitions of state, control input, and disturbance input vectors:

$$
\begin{aligned}
X_{j} & =\left[\begin{array}{llll}
x_{1}^{j-1} x_{2}^{j-2} & \cdots & x_{N}^{j-N}
\end{array}\right]^{T} \\
U_{j} & =\left[\begin{array}{lll}
u_{0}^{j} u_{1}^{j-1} & \cdots & u_{N-1}^{j-N+1}
\end{array}\right]^{T} \\
W_{j} & =\left[\begin{array}{llll}
w_{0}^{j} w_{1}^{j-1} & \cdots & w_{N-1}^{j-N+1}
\end{array}\right]^{T} .
\end{aligned}
$$

We notice that the state vector $X_{j}$ is made up of the time deviations $x_{k}^{i}$ such that the sum of their indexes is precisely equal to the state index $j: X_{j}=\left\{x_{k}^{i} \mid i+k=j\right\}$.

It then follows from the basic traffic dynamics equation (7) that $X_{j+1}$ is generated by $X_{j}$ (i.e., $x_{k+1}^{i}$ is generated by $x_{k}^{i}$ and $x_{k+1}^{i-1}$ for all $\langle i\rangle$ and $\langle k\rangle$ ). This is expressed in the compact matrix form by the following state-space model:

$$
X_{j+1}=A X_{j}+B U_{j}+B W_{j}
$$

with

$$
\begin{gathered}
A=D 2\left(N ;-\frac{c_{1}}{1-c_{1}}, \cdots,-\frac{c_{N}}{1-c_{N}}\right. \\
\left.\frac{1}{1-c_{2}}, \cdots, \frac{1}{1-c_{N}}\right) \\
B=D 1\left(N ; \frac{1}{1-c_{1}}, \cdots, \frac{1}{1-c_{N}}\right) .
\end{gathered}
$$


Performance Index: For a given $X_{j}$, the control law $U$ is computed in order to minimize, under the linear constraint (9), the following criterion:

$$
\begin{aligned}
J_{1}=p X_{j+1}^{T} X_{j+1}+q\left(X_{j+1}-X_{j}\right)^{T}\left(X_{j+1}\right. & \left.-X_{j}\right) \\
& +U_{j}^{T} U_{j}
\end{aligned}
$$

where $p$ and $q$ are two positive constant design variables at the user's disposal. The motivation for this criterion is as follows. The first term $X_{j+1}^{T} X_{j+1}$ penalizes the deviations with respect to the nominal schedule while the second term penalizes the deviations of the intervals between successive trains from the nominal value $(H)$ and is therefore related to the average waiting time for the passengers at a platform. This term may be used to enhance the traffic regularity, irrespective of the nominal time table. The third term penalizes control actions that are too large. The values of $p$ and $q$ depend on the control purpose and reflect a trade-off between the regulation objectives (i.e., time table versus regularity). The choice of the criterion (10) and of the specialized statespace formulation ( 9 ) is primarily justified by the fact that their combination leads to a very simple, meaningful, and stabilizing control law, as we shall now demonstrate.

Feedback Control Law: The control minimizing (10) under constraint (9) is linear in $X_{j}$ and is written

$U_{j}=-\left[I_{N}+(p+q) B^{T} B\right]^{-1}\left[(p+q) B^{T} A-q B^{T}\right] X_{j}$

where $I_{N}$ denotes the identity matrix of dimension $N$. The components of $U_{j}$ can also be expressed as follows:

$$
\begin{aligned}
& u_{k}^{i}=-g_{k+1} x_{k}^{i}+f_{k+1} x_{k+1}^{i-1} \\
& \text { where } \quad g_{k+1}=\frac{p+q}{p+q+\left(1-c_{k+1}\right)^{2}} \quad \text { and } \\
& f_{k+1}=\frac{q+p c_{k+1}}{p+q+\left(1-c_{k+1}\right)^{2}} \text {. }
\end{aligned}
$$

This means that the control applied to train $\langle i\rangle$ between platforms $\langle k\rangle$ and $\langle k+1\rangle$ is a linear combination of two time deviations: the time deviation of train $\langle i\rangle$ at platform $\langle k\rangle$ and the time deviation of the preceding train $\langle i-1\rangle$ at the next platform $\langle k+1\rangle$.

Closed-Loop Stability: The state matrix of the closed-loop system corresponding to the state-space formulation (9) coupled with the feedback control law (11) is easily seen to be exponentially stable. The closed-loop system is therefore "bounded-input bounded-state" stable. On the other hand, it is more interesting, from a practical point of view, to examine the variations of the mean-square value of the time deviations at a given platform in the closed-loop system. In absence of disturbances, the time deviations at two successive platforms are related by the following nonstationary closedloop equation:

$$
X_{k+1}^{S}=\bar{A}_{k}^{S} X_{k}^{S}
$$

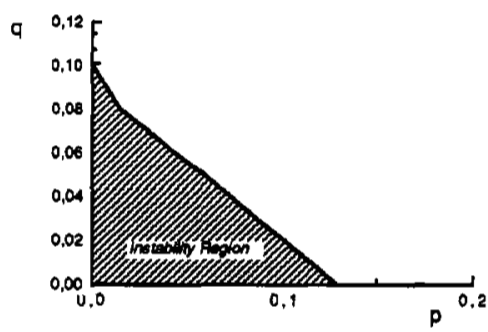

Fig. 4. Instability region for $c_{k+1}=0.1$.

where $\quad \bar{A}_{k}^{S}=\bar{A}_{k}^{-1} B_{k} \quad$ is lower triangular with eigenvalues

$$
\begin{gathered}
\qquad\left(1-g_{k+1} / 1-c_{k+1}\right), \\
\bar{A}_{k}=D 2\left(N ; 1-c_{k+1} ; f_{k+1}+c_{k+1}\right) \text { and } \\
\bar{B}_{k}=D 1\left(N ; 1+g_{k+1}\right) .
\end{gathered}
$$

Since the model (12) is not stationary, no definite general conclusion can be drawn immediately from the structure of the matrix $\bar{A}_{k}^{S}$ concerning the evolution of the mean-square deviation at a given platform. We can, however, state the following result.

Theorem 2: For $p$ and $q$ such that

1) $(p+q)>c_{k+1}\left(1-c_{k+1}\right)$

2) $\left|c_{k+1}\left(1-c_{k+1}\right)-q\right|$

$$
<\frac{\left[p+q+\left(1-c_{k+1}\right)\right]^{2}-\left(1-c_{k+1}\right)^{2}}{p+q+\left(1-c_{k+1}\right)^{2}}
$$

the mean-square value of the time deviations at any platform decreases exponentially from platform to platform.

This theorem is easily proved by using similar arguments as for Theorem 1. The conditions (13) are satisfied for a wide range of positive $p$ and $q$. To illustrate this, the domain of the $(p, q)$ plane wherein these conditions are achieved, for $c_{k+1}=0.1$, is represented in Fig. 4 .

Comment: The simple one-step-ahead performance index $(10)$ is attractive because, under the constraint (9), it leads to a simple stabilizing control law. We could also consider a multistep index of the following form:

$$
\begin{aligned}
J=\sum_{1=1}^{N}\left\{p X_{j+1}^{T} X_{j+1}+q\left(X_{j+1}-X_{j+1-1}\right)^{T}\right. \\
\left.\cdot\left(X_{j+1}-X_{j+1-1}\right)+U_{j+1-1}^{T} U_{j+1-1}\right\} .
\end{aligned}
$$

The corresponding optimal control law will be linear in the state vector $X_{j}$, with a gain matrix obtained from the solution of a Riccati equation. In this case, the control to be applied to train $\langle i\rangle$ at platform $\langle k\rangle$ involves all the time deviations $x_{k}^{i}$ relative to index $j$ such that $i+k=j$.

\section{TRAFFIC ANALYSIS FOR LOOP LINES}

This section deals with the description and control of the traffic on loop lines whereon the sequence of platforms 
crossed by every train is periodic. We consider a set of $M$ trains which are operated simultaneously on a loop line with $N$ platforms $(M<N)$ (see Fig. 2). Without traffic control, the time deviations with respect to a nominal operation are cumulated from circuit to circuit. Currently, on most commercial loop lines, traffic control consists only of resetting the time deviations to zero at selected platforms (generally the terminus stations) by use of time margins. This solution, however, requires operation of more trains than necessary and consequently, at each instant, several trains are in standby. The aim of this section is then to design efficient traffic control laws which are adapted to the periodic structure of the line, which ensure the stability of the operation, do not need time margins and allow therefore to operate a minimal number of trains.

We first introduce in Section V-A the concept of natural interval between successive trains which characterizes the nominal operation of a loop line. In Section V-B, we show how the traffic description w.r.t. a nominal time schedule has to be modified in order to account for the periodic structure of the line. Then, we design in Section V-C a generalized performance index specific for loop lines which allows us to compute a stabilizing control law having the same properties as that for open lines. In Section V-D, we extend the traffic description when there is no reference schedule and in Section V-E we design traffic ' control algorithms valid for this situation. Finally, Section V-F presents an attractive solution incorporating estimation to cope with the possibly inaccurate knowledge of the natural interval.

To simplify the presentation of the following sections, we make the following additional assumption.

A5: The $c_{k}$ are identical for all platforms, i.e., $c_{k}=c$, $k=1, \cdots, N$.

\section{A. Natural Interval Between Successive Trains}

The objective is to control the traffic regularity, i.e., to maintain the line at a nominal steady-state situation characterized by a constant time interval $H$ between successive trains. It must be pointed out that for a given loop line (characterized by $S, R_{k}, c_{k}$ ) with a given number of trains, this nominal interval cannot be chosen arbitrarily (unlike open lines). To see this, consider a loop line operated at the nominal situation, with a constant time interval $H$ between successive trains. The transfer time for a full circuit, i.e., the trip time of any train between two successive departures from the same platform of the line, is obviously equal to $M$ (number of trains) times $H$. Indeed, between two successive departures of a train from a platform, all the other trains operated on the line have also left this platform, each separated by the time interval $H$. But, on the other hand, this transfer time is also equal to the sum of the transfer times between the $N$ platforms and the staying times at the platforms modeled by (3). Hence

$$
M H=N[S+c]+\sum_{k=1}^{N} R_{k} .
$$

For a given line, the value of $H$ satisfying (14) is called "the natural interval."

\section{B. Traffic Description with Reference to a Nominal Schedule}

The basic dynamical equation (4) holds for loop lines, provided the two indexes $\langle i\rangle$ and $\langle k\rangle$ are interpreted, respectively, modulo $M$ and modulo $N$ : $\operatorname{train}\langle M\rangle$ is followed by train $\langle 1\rangle$ and platform $\langle N\rangle$ by platform $\langle 1\rangle$. With this interpretation, Equation (6), where $H$ is the natural interval, defines the nominal time table. The perturbation equation ( 7 ) holds also for loop lines. However, the combination of these scalar equations in order to define a global state-space formulation must reflect the periodicity of the structure.

Operation Quality Index: We define

$$
\Pi_{k}=\frac{1}{M} \sum_{i=1}^{M}\left(x_{k-i}^{i}\right)^{2}
$$

as a quality index for loop lines. This index is defined as the mean-square value of the time deviations of the $M$ operating trains (indexes $\langle 1\rangle$ to $(M\rangle)$ at $M$ successive platforms (indexes $(k-1\rangle$ to $\langle k-M\rangle$, modulo $N)$. Notice that this index is equal to zero in nominal operation.

Matrix Notation: Before defining a convenient state-space formulation to study the traffic instability properties of loop lines, we introduce the following matrix notation.

$D_{3}(a, b)$ is an $N \times N$ matrix of the following form:

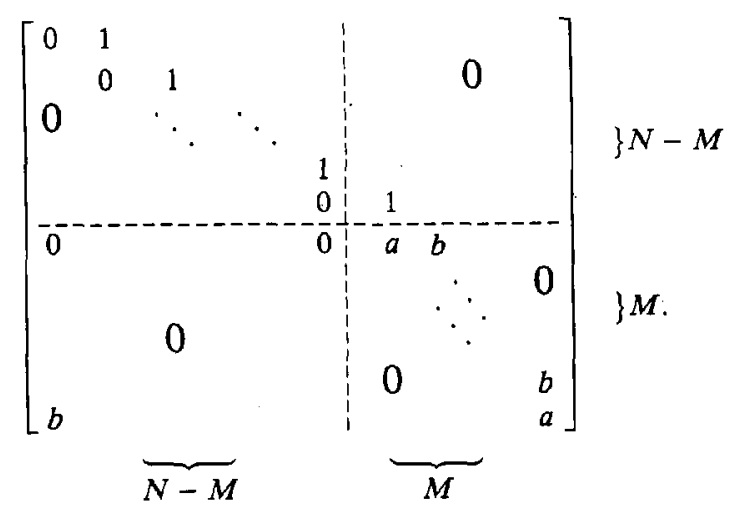

This matrix is parametrized by the real values $a$ and $b$. Its characteristic polynomial is $\rho(\lambda)=\lambda^{N-M}(\lambda-a)^{M}-b^{M}$. $D_{4}(a)$ denotes an $N \times M$ matrix of the following form:

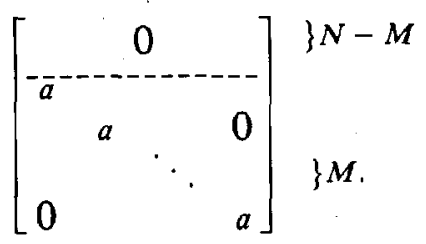

Intrinsic Instability of Loop Lines: For each train $\langle i\rangle$, we define, respectively, as state, control, and disturbance 
vectors

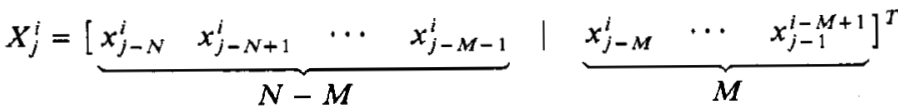

$$
\begin{aligned}
& U_{j}^{i}=\left[\begin{array}{llll}
u_{j-M}^{i} & u_{j-M+1}^{i-1} & \cdots & u_{j-1}^{i-M+1}
\end{array}\right]^{T} \\
& W_{j}^{i}=\left[\begin{array}{llll}
w_{j-M}^{i} & w_{j-M+1}^{i-1} & \cdots & w_{j-1}^{i-M+1}
\end{array}\right]^{T}
\end{aligned}
$$

where $i \in\{1,2, \cdots, M\}$. The state vector $X_{j}^{i}$, associated with train $\langle i\rangle$, is the $N$-vector whose components have the following meaning.

1) The $(N-M)$ first components are the time deviations of train $\langle i\rangle$ at $(N-M)$ successive platforms (index $\langle j-N\rangle$ to $\langle j-M-1\rangle)$.

2) The last $M$ components are the time deviations relative to the $M$ trains and characterized by the same value $j$ for the sum of their upper and lower indexes (interpreted, as usual, respectively, modulo $M$ and modulo $N$ ).

The control and disturbance vectors $\left(U_{j}^{i}\right.$ and $\left.W_{j}^{i}\right)$ are $M$-vectors whose components are relative to the $M$ trains and characterized by the same value $j$ for the sum of their upper and lower indexes. With the definitions (16), we obtain from the basic equation (7), the following state-space formulation:

$$
X_{j+1}^{i}=A_{L} X_{j}^{i}+B_{L}\left[U_{j}^{i}+W_{j}^{i}\right], \quad i \in\{1,2, \cdots, M\}
$$

where

$$
A_{L}=D_{3}\left(\frac{1}{1-c}, \frac{-c}{1-c}\right) \text { and } B_{L}=D_{4}\left(\frac{1}{1-c}\right) .
$$

Remark: It follows from (16) and (17) that for a loop line with $M$ operating trains there exist $M$ equivalent state-space representations with identical state-space matrices. Moreover, it must be noticed that the values of $\Pi_{k}$ for two successive circuits can be related using (16), i.e.,

$$
\Pi_{k+N}-\Pi_{k}=\frac{1}{M} \sum_{i=1}^{M}\left[\left\|X_{j+1}^{i}\right\|^{2}-\left\|X_{j}^{i}\right\|^{2}\right]
$$

The free system, without disturbances and control actions, associated with (17) is written $(i \in\{1, \cdots, M\})$

$$
X_{j+1}^{i}=A_{L} X_{j}^{i} \text {. }
$$

For $c=0$, the eigenvalues of $A_{L}$ are 0 and 1 , whose algebraic multiplicity $(M)$ is equal to the geometric multiplicity. This implies that the free system is stable but not asymptotically. There is therefore no guarantee of boundedness of the solutions in the presence of bounded disturbances. For $c>0$, one eigenvalue is equal to 1 , with a corresponding eigenvector of the form $[1 \cdots 1]^{T}$. The situation where all the deviations have the same value (i.e., a state vector of the form $\alpha[1 \cdots 1]^{T}$, for any $\alpha$ ) is therefore an equilibrium which is however not stable: for any perturbation affecting the state vector, its norm as well as the index $\Pi_{k}$ tend to infinity.

Theorem 3: For $c>0$, for any perturbation of the equilibrium, there exists $j_{0}(\in N)$ and $l_{0}(\in N)$ such that $\forall i$ $(i=1, \cdots, M), \forall k(k=1, \cdots, N), \forall j \geq j_{0}, \forall l \geq l_{0}$

1) $\left\|X_{j+1}^{i}\right\|>\left\|X_{j}^{i}\right\|$

2) $\Pi_{k+(l+1) * N}>\Pi_{k+i * N}$.

Proof: See the Appendix, Section A.2.

This result illustrates the natural instability of a loop line and justifies the need for traffic control strategies.

\section{Traffic Control with a Nominal Time Schedule}

As for the open line case (Section IV), the feedback control is designed in order to minimize a quadratic performance index, under the linear constraint (17).

Performance Index: By analogy with the performance index of open lines $J_{1}(10)$, we define the following performance criterion:

$J_{2}^{i}=\left(X_{j+1}^{i}\right)^{T} P\left(X_{j+1}^{i}\right)+\left(X_{j+1}^{i}-S_{N} X_{j}^{i}\right)^{T}$

$$
\cdot Q\left(X_{j+1}^{i}-S_{N} X_{j}^{i}\right)+\left(U_{j}^{i}\right)^{T} U_{j}^{i}
$$

where

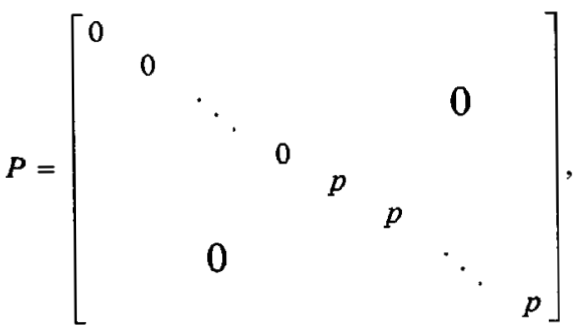<smiles>O=S(=O)(O)O[Na]</smiles>

$P$ and $Q$ are diagonal weighting matrices of dimension $N$ whose $(N-M)$ first components are equal to zero. The remaining components are equal, respectively, to $p$ and $q$ which are two nonnegative design parameters. $S_{N}$ is the 
$N \times N$ permutation matrix

$$
S_{N}=\left[\begin{array}{cccc}
0 & 1 & & 0 \\
& \ddots & \ddots & \\
0 & & & 1 \\
1 & & & 0
\end{array}\right] .
$$

The first term of $J_{2}^{i}$ penalizes the $M$ time deviations of the $M$ operated trains and the second term, as in $J_{1}$, the $M$ interval deviations from the nominal value $H$ between successive trains. The third term penalizes control actions that are too large.

Remark: $J_{1}$ accounts for $N$ (number of platforms) time deviations while $J_{2}^{i}$ accounts for $M$ (number of trains) time deviations. This reflects a structural difference between open and loop lines. Indeed, an open line can be conceptually seen as a line with a finite number $(N)$ of platforms and an infinite number of operated trains while a loop line can be represented with a finite number $(M)$ of operating trains and an infinite number of platforms obtained by the periodic repetition of their initial sequence.

Feedback Control Law: The control minimizing (19) under the linear constraint (17) is linear in $X_{j}^{i}$

$$
\begin{aligned}
& U_{j}^{i}=\left[I_{M}+B_{L}^{T}(P+Q) B_{L}\right]^{-1} \\
& \cdot\left[B_{L}^{T}(P+Q) A_{L}-B_{L}^{T} Q S_{N}\right] X_{j}^{l} .
\end{aligned}
$$

The components of $U_{j}^{i}$ can also be expressed as follows:

$$
u_{k}^{i}=g x_{k}^{i}+f x_{k+1}^{i-1}
$$

where $g=(p+q) /\left[p+q+(1-c)^{2}\right]$ and $f=-(p c$ $+q) /\left[p+q+(1-c)^{2}\right]$. This control law has the same form as for the open line case: the control to be applied to a given train $\langle i\rangle$ at a given platform $\langle k\rangle$ is a linear combination

- of the time deviation of this train $\langle i\rangle$ at the same platform $\langle k\rangle$ (weighting coefficient $g$ );

- of the time deviation of the preceding train $\langle i-1\rangle$ at the next platform $\langle k+1\rangle$ (weighting coefficient $f$ ).

Closed-Loop Behavior: With the state feedback (20), the closed-loop behavior is described by the following equation:

$$
X_{j+1}^{i}=\bar{A}_{L} X_{j}^{i}+B_{L} W_{j}^{i}, \quad i \in\{1,2, \cdots, M\}
$$

where $\bar{A}_{L}=D_{3}[(1-g) /(1-c),-(c+f) /(1-c)]$ and $B_{L}=D_{4}[1 /(1-c)]$. From (22), we can state the following result.

Theorem 4: For $q>c(1-c)$, at any platform $\langle k\rangle$

1) if $p>0$, all the eigenvalues of $\bar{A}_{L}$ are strictiy inside the unit circle;

2) if $p=0$, all the eigenvalues are strictly inside the unit circle except one, equal to 1 , with a corresponding eigenvector of the form $[1 \cdots 1]^{T}$.

Proof: See the Appendix, Section A.3.

For $p>0$, this result implies the asymptotic stability of the closed-loop system and therefore the monotonic convergence to zero of the mean-square deviation $\Pi_{k}$ :

$$
\forall k>0, \quad \Pi_{k+N} \leq \Pi_{k} \text { and } \lim _{1 \rightarrow \infty} \Pi_{k+1^{*} N}=0 .
$$

For $p=0$, the solution of the closed-loop system converges, in the absence of disturbances, to a steady-state situation characterized by a constant time deviation with respect to the nominal schedule, i.e.,

$$
\exists \lambda \text { such that } \lim _{1 \rightarrow \infty} \Pi_{k+1^{*} N}=\lambda^{2} .
$$

This means that if the interval regulation $(p=0)$ is the only control objective, a nominal time schedule is no longer necessary, as we shall see in the next section.

\section{Traffic Description Without Reference to a Nominal Time Schedule}

So far, in the preceding section, we have analyzed the traffic dynamics in terms of time deviations between the actual system behavior and a given nominal schedule. On the other hand, it is clear that a nominal schedule is not really required for fully automated high-density lines. In this section, we show how to carry out the description and the analysis of the traffic in this case.

Interval Equation: We define $y_{k}^{i} \triangleq t_{k}^{i}-t_{k}^{i-1}-H$ as the deviation, with respect to $H$, of the nominal time interval between trains $\langle i\rangle$ and $\langle i-1\rangle$ at platform $\langle k\rangle$. Thus, from (4), the traffic dynamics are rewritten as follows:

$$
\begin{aligned}
(1-c) y_{k+1}^{i}+c y_{k+1}^{i-1}=y_{k}^{i}+\delta u_{k}^{i}+\delta w_{k}^{i}, & \\
k & \geq 1, \quad i \geq 1
\end{aligned}
$$

with $\delta u_{k}^{i} \triangleq u_{k}^{i}-u_{k}^{i-1}$ and $\delta w_{k}^{i} \triangleq w_{k}^{i}-w_{k}^{i-1}$. Equation (23) is called "interval equation." It holds for loop lines, provided the two indexes $\langle i\rangle$ and $\langle k\rangle$ are interpreted, respectively, modulo $M$ and modulo $N$. This equation is initialized with $y_{k}^{0}=0, \forall_{k}$ and $\delta u_{k}^{1}=\delta w_{k}^{1}=0, \forall k$.

State-Space Formulation: Similarly to Section V-B, we define, respectively, as state, control, and disturbance vectors:

$$
\begin{gathered}
Y_{j}^{i}=[\underbrace{y_{j-N}^{i} y_{j-N+1}^{i} \cdots y_{j-M-1}^{i}}_{N-M} \mid \underbrace{y_{j-M}^{i} \cdots y_{j-1}^{i-M+1}}_{M}]^{T} \\
\Delta U_{j}^{i}=[\underbrace{\delta u_{j-M}^{i} \delta u_{j-M+1}^{i-1} \cdots \delta u_{j-1}^{i-M+1}}_{M}]^{T} \\
\Delta W_{j}^{i}=[\underbrace{\delta w_{j-M}^{i} \delta w_{j-M+1}^{i-1} \cdots \delta w_{j-1}^{i-M+1}}_{M}]^{T}
\end{gathered}
$$

and we obtain from the interval equation (23), the following state-space formulation:

$$
Y_{j+1}^{i}=A_{L} Y_{j}^{i}+B_{L}\left(\Delta U_{j}^{i}+\Delta W_{j}^{i}\right), \quad i \in\{1, \cdots, M\}
$$

where $A_{L}=D_{3}[1 /(1-c),-c /(1-c)]$ and $B_{L}=$ $D_{4}[1 /(1-c)]$. Again, due to the loop structure, there exist $M$ equivalent state-space formulations with identical statespace matrices and which can be distinguished by the upper index of $Y_{j}^{i}, \Delta U_{j}^{i}$, or $\Delta W_{j}^{i}$.

In order to describe the evolution of the control actions and of the disturbances, we define two augmented $N$-vectors $\bar{U}_{j}^{i}$ 
and $\bar{W}_{j}^{i}$ whose $M$ last components coincide with the $M$ components of $U_{j}^{i}$ and $W_{j}^{i}$ defined by (16)

$$
\begin{gathered}
\bar{U}_{j}^{i}=[\underbrace{u_{j-N}^{i} u_{j-N+1}^{i} \cdots u_{j-M-1}^{i}}_{N-M} \mid \underbrace{u_{j-M}^{i} \cdots u_{j-1}^{j-M+1}}_{M}]^{T} \\
\bar{W}_{j}^{i}=[\underbrace{w_{j-N}^{i} w_{j-N+1}^{i} \cdots w_{j-M-1}^{i}}_{N-M} \mid \underbrace{\mid w_{j-M}^{i} \cdots w_{j-1}^{j-M+1}}_{M}]^{T} .
\end{gathered}
$$

The evolution of $\bar{U}_{j}^{i}$ and $\bar{W}_{j}^{i}$ is then described by

$$
\begin{aligned}
\bar{U}_{j}^{i}=S_{N} \bar{U}_{j-1}^{i}+D_{4}(1) \Delta U_{j}^{i} ; & i \in\{1, \cdots, M\} \\
\bar{W}_{j}^{i}=S_{N} \bar{W}_{j-1}^{i}+D_{4}(1) \Delta W_{j}^{i} ; & i \in\{1, \cdots, M\}
\end{aligned}
$$

with $S_{N}$ defined in (19). The vectors $Y_{j}^{i}, \bar{U}_{j}^{i}, \bar{W}_{j}^{i}$ are not independent: they are related by a linear compatibility condition as stated in the following lemma.

Lemma 1: For any $j$ and for any $i \in\{1, \cdots, M\}, Y_{j}^{i}$ $\bar{U}_{j-1}^{i}$ and $\bar{W}_{j-1}^{i}$ satisfy

$$
b_{1}^{T} Y_{j}^{i}+b_{2}^{T}\left(\bar{U}_{j-1}^{i}+\bar{W}_{j-1}^{i}\right)=0
$$

where $b_{1}$ and $b_{2}$ are the following $N$-vectors:

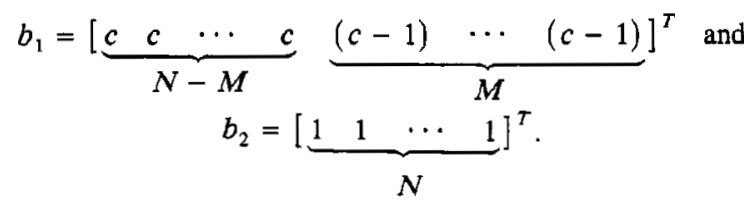

Proof: See the Appendix, Section A.4.

Setpoint: The operational objective is to keep the time interval between any pair of successive trains as close as possible to the natural interval $H$. The corresponding desired setpoint is therefore defined by

$$
\forall i \in\{1, \cdots, M\}, \quad \forall j, \quad Y_{j}^{i}=\bar{U}_{j-1}^{i}=0 .
$$

Operation Quality Index: We define

$$
\sigma_{k}=\frac{1}{M} \sum_{i=1}^{M}\left(y_{k-i}^{i}\right)^{2}
$$

as a quality index for loop lines when the goal is to ensure the regularity between successive trains. This index is defined as the mean-square value of the $M$ deviations, with respect to the natural interval $H$ of the nominal interval, corresponding to the $M$ operating trains (indexes $\langle 1\rangle$ to $\langle M\rangle$ ) arranged in pairs at $M$ successive platforms (indexes $\langle k-1\rangle$ to $\langle k-$ $M)$ ). This index is equal to zero at the setpoint which, however, is unstable as shown hereafter in Theorem 5. As for the $\Pi_{k}$ index, the evolution of $\sigma_{k}$ from circuit to circuit is related to the evolution of the state vectors $Y_{j}^{i}$

$$
\sigma_{k+N}-\sigma_{k}=\frac{1}{M} \sum_{i=1}^{M}\left[\left\|Y_{j+1}^{i}\right\|^{2}-\left\|Y_{j}^{i}\right\|^{2}\right] .
$$

Intrinsic Instability: Consider the free system corresponding to (25). As pointed out in Section V-B, for $c>0$, $A_{L}$ has an eigenvalue equal to 1 , with a corresponding eigenvector of the form $[1 \cdots 1]^{T}$. This means that the situation where all the components of $Y_{j}^{i}$ are equal, say to $\alpha$, is an equilibrium point. From the compatibility condition
(28), the corresponding $\left[\bar{U}_{j}^{i}+\bar{W}_{j}^{i}\right]$ is a vector whose elements are all equal to $\alpha(N-M-c) / N$. Each of these equilibrium points (characterized by $\alpha$ ) corresponds to a constant time interval between successive trains, but which is different from $H$. Furthermore, these equilibrium points are unstable, as stated in the following theorem.

Theorem 5: For $c>0$, for any perturbation (compatible with (24)), there exists $j_{0}(\in N)$ and $l_{0}(\in N)$ such that

$$
\begin{gathered}
\forall i(i=1, \cdots, M), \quad \forall k(k=1, \cdots, N), \\
\forall j \geq j_{0}, \quad \forall l \geq l_{0}
\end{gathered}
$$

1) $\left\|Y_{j+1}^{i}\right\|>\left\|Y_{j}^{i}\right\|$

2) $\sigma_{k+(l+1)^{*} N}>\sigma_{k+l^{*} N}$.

Proof: Similar to the proof of Theorem 3.

\section{E. Traffic Control Without Nominal Time Schedule}

Once again, the feedback control is designed in order to minimize a quadratic performance index.

Performance Index: The following performance index is considered:

$$
J_{3}^{i}=\left(Y_{j+1}^{i}\right)^{T} Q Y_{j+1}^{i}+\left(\bar{U}_{j}^{i}\right)^{T} \bar{U}_{j}^{i}
$$

where $Q$ is the same diagonal weighting matrix of dimension $N$ as those defined in $J_{2}^{i}(19)$. The two terms of $J_{3}^{i}$ penalize, respectively, the deviations of $Y_{j+1}^{i}$ and $\bar{U}_{j}^{i}$ from the setpoint.

Feedback Control Law: The control minimizing (29) under the linear constraints (25) and (27a) is linear in $Y_{j}^{i}$ and $\bar{U}_{j-1}^{i}$

$$
\begin{gathered}
\Delta U_{j}^{i}=K_{1} Y_{j}^{i}+K_{2} \bar{U}_{j}^{i} \\
\text { where } K_{1}=-\left[B_{L}^{T} Q B_{L}+I_{M}\right]^{-1} B_{L}^{T} Q A_{L} \\
K_{2}=-\left[B_{L}^{T} Q B_{L}+I_{M}\right]^{-1}\left[D_{4}(1)\right]^{T} S_{N}
\end{gathered}
$$

where $I_{M}$ is the identity matrix of dimension $M$. The control action to be applied to train $\langle i\rangle$ at platform $\langle k\rangle$ is therefore given by

$$
\begin{aligned}
& u_{k}^{i}=(1+h) u_{k}^{i-1}+g y_{k}^{i}+f y_{k+1}^{i-1} \\
& \text { where } f=\frac{q c}{q+(1-c)^{2}}, \quad g=\frac{-q}{q+(1-c)^{2}} \text {, } \\
& h=\frac{-(1-c)^{2}}{q+(1-c)^{2}} \text {. }
\end{aligned}
$$

This control action is a linear combination of:

- the control action applied to the preceding train $\langle i-1\rangle$ at the same platform $\langle k\rangle$ (weighting coefficient $1+h$ );

- the time deviation w.r.t. the natural interval of the time interval between trains $\langle i\rangle$ and $\langle i-1\rangle$ measured at the same platform $\langle k\rangle$ (weighting coefficient $g$ );

- the time deviation w.r.t. the natural interval of the time interval between trains $\langle i-1\rangle$ and $\langle i-2\rangle$ measured at the next platform $\langle k+1\rangle$ (weighting coefficient $f$ ).

Remark: It is interesting to notice that this control law 
coincides with the control law (21), with $p=0$, provided that a reference time schedule compatible with the natural interval $H$ is used. Indeed, in that case, we have

$$
y_{k}^{i}=t_{k}^{i}-t_{k}^{i-1}-H=x_{k}^{i}-x_{k}^{i-1}
$$

and (31) can be rewritten as

$$
\begin{aligned}
u_{k}^{i}= & \frac{q}{q+(1-c)^{2}}\left[x_{k}^{i-1}-x_{k}^{i}\right]+\frac{q}{q+(1-c)^{2}} \\
& \cdot\left[u_{k}^{i-1}+x_{k}^{i-1}-c x_{k+1}^{i-2}+(c-1) x_{k+1}^{i-1}\right] \\
= & \frac{q}{q+(1-c)^{2}}\left[x_{k}^{i-1}-x_{k}^{i}\right]
\end{aligned}
$$

which is the control deducted from (23) for $p=0$.

Closed-Loop Behavior: With the feedback control (30) the closed-loop behavior is described by

$$
\begin{array}{r}
{\left[\begin{array}{c}
Y_{j+1}^{i} \\
\bar{U}_{j}^{i}
\end{array}\right]=\left[\begin{array}{cc}
A_{L}+B_{L} K_{1} & B_{L} K_{2} \\
0_{N-M, M} & {\left[S_{N}+0_{N-M, M}\right]} \\
K_{1} & K_{2}
\end{array}\right]\left[\begin{array}{c}
Y_{j}^{i} \\
\bar{U}_{j-1}^{i}
\end{array}\right]} \\
+\left[\begin{array}{c}
B_{L} \\
0_{N-M, M} \\
I_{M}
\end{array}\right] \Delta W_{j}^{i}
\end{array}
$$

where $0_{N-M . M}$ denotes the $(N-M) \times M$ null matrix. Consider first the disturbance free case, i.e., $W_{j}^{i}=0$ and $\Delta W_{j}^{i}=0$, for each $j$ and for each $i$. We have the following result.

Theorem 6: For $W_{j}^{i}=0$ and $\Delta W_{j}^{i}=0$, for each $j$ and for each $i \in\{1, \cdots, M\}$ and for trajectories $\left(Y_{j}^{i}, U_{j}^{i}\right) \mathrm{com}-$ patible with the line structure in the sense of $(28)$, the feedback control law (30) with $q>c(1-c)$ guarantees

1) that the operation quality index $\sigma_{k}$ is decreasing asymptotically and converges to zero from circuit to circuit

$$
\begin{gathered}
\forall k \in\{\langle 1\rangle, \cdots,\langle N\rangle\}, \forall l>0, \quad \sigma_{k+l^{* N}} \leq \sigma_{k} \quad \text { and } \\
\lim _{l \rightarrow \infty} \sigma_{k+l^{*} N}=0
\end{gathered}
$$

2) that the corresponding control converges asymptotically to zero

$$
\lim _{j \rightarrow \infty} U_{j}^{i}=0 \text {. }
$$

Proof: See the Appendix, Section A.5.

This result can be exploited to characterize the structure of the eigenvalues and the eigenvectors of the closed-loop matrix (32) by the following lemma.

Lemma 2: If $b$ is the vector $b^{T}=\left[b_{1} b_{2}\right]^{T}$ defined by (28), then the closed-loop matrix defined in (32) with $q>$ $c(1-c)$

1) has an eigenvalue equal to one. The corresponding eigenvector is of the form $[-(1-c) / q, \cdots,-(1-c) / q$, $1, \cdots, 1]^{T}$ which is not orthogonal to the vector $b$;

2) the other eigenvalues are strictly inside the unit circle and the corresponding eigenvectors are orthogonal to $b$.

Proof: See the Appendix, Section A.6.

These technical results allow us to prove the boundedness of the operation quality index $\sigma_{k}$ in the presence of bounded disturbances.

Theorem 7: For $q>c(1-c)$, in presence of bounded disturbances, the feedback control law (30) ensures the boundedness of the operation quality index $\sigma_{k}$ and of the corresponding control vector.

Proof: See the Appendix, Section A.7.

\section{F. Unknown Natural Interval}

The implementation of the feedback control law (30) requires an accurate knowledge of the natural interval $H$. On the other hand, as the number of operating trains on the line varies during an operation day, the value of the natural interval may change. Therefore, in this section, we first investigate the influence on the control performance of a modeling. error on $H$. Then, we present an asymptotic estimator designed in order to track the value of the natural interval $H$ when the number of trains is changing. This estimation, combined with the control law (30) guarantees closed-loop convergence.

Influence of a Modeling Error: Assume that the natural interval $H$ is approximated by $\hat{H}$ and define the offset $\tilde{H}$ as

$$
\tilde{H}=H-\hat{H}
$$

Defining $\hat{Y}_{j}^{i}$ to be the estimate of $Y_{j}^{i}$, we have

$$
\hat{Y}_{j}^{i}=Y_{j}^{i}+\tilde{H} b_{2}
$$

where $b_{2}$ is the $N$ vector (28). Then, the components of $\hat{Y}_{j}^{i}$ denoted $\hat{y}_{k}^{i}$ are $\hat{y}_{k}^{i}=t_{k}^{i}-t_{k}^{i-1}-\hat{H}=y_{k}^{i}+\hat{H}$. The control law (30) is implemented with the estimate $\hat{Y}_{j}^{i}$

$$
\Delta U_{j}^{i}=K_{1} \hat{Y}_{j}^{i}+K_{2} \bar{U}_{j}^{i} .
$$

Theorem 8: In the absence of disturbances, the system defined by (25)-(27) coupled with the control law (35) converges to an equilibrium point characterized by

- a steady-state interval $H^{*}$

$$
\begin{aligned}
H^{*}=H & \frac{(1-c)(N c-M)}{(1-c)(N c-M)-q M} \\
& -\hat{H} \frac{q M}{(1-c)(N c-M)-q M} ;
\end{aligned}
$$

- a steady-state control $u^{*}$

$$
u^{*}=-(H-\hat{H}) q \frac{(N c-M)}{(1-c)(N c-M)-q M} .
$$

Proof: See the Appendix, Section A.8.

When $H=\hat{H}$, this equilibrium point is the desired setpoint $Y_{j}^{i}=0, \bar{U}_{j}^{i}=0$ ). In the presence of bounded disturbances it can easily be shown, as in Theorem 7, that the deviations with respect to the equilibrium point $\left(H^{*}, u^{*}\right)$ are bounded.

Estimation of the Natural Interval H: The natural interval $H$ can be estimated recursively. Let $\hat{H}_{j}$ be the estimation used in the implementation of the control law, i.e., 


$$
\begin{aligned}
& \hat{Y}_{j}^{i}=Y_{j}^{i}+\tilde{H}_{j} b_{2} . \text { Then, using (28) and (34) we obtain } \\
& \begin{aligned}
b_{1}^{T} \hat{Y}_{j}^{i}+b_{2}^{T} \bar{U}_{j-1}^{i} & =b_{1}^{T} b_{2} \tilde{H}_{j}-b_{2}^{T} \bar{W}_{j-1}^{i} \\
& =(c N-M) \tilde{H}_{j}-b_{2}^{T} \bar{W}_{j-1}^{i} .
\end{aligned}
\end{aligned}
$$

This suggests the following estimator of $H$ :

$$
\hat{H}_{j+1}=\hat{H}_{j}+\alpha\left[b_{1}^{T} \hat{Y}_{j}^{i}+b_{2}^{T} \bar{U}_{j-1}^{i}\right]
$$

where $\alpha$ is a design parameter. The estimation error dynamics are written

$$
\tilde{H}_{j+1}=[1-\alpha(c N-M)] \tilde{H}_{j}+\alpha b_{2}^{T} \bar{W}_{j-1}^{i} .
$$

For $\alpha$ chosen such that

$$
|1-\alpha(c N-M)|<1
$$

the estimator is asymptotically stable, independent of the control law. This is summarized in the following theorem.

Theorem 9: The control law (35) with $\hat{Y}_{j}^{i}$ and the adaptation law (36) for $\hat{H}_{j}$ with $\alpha$ satisfying (38) ensures the asymptotic stability of the closed-loop system around $Y_{j}^{i}=0$, $\bar{U}_{j}^{i}=0$, and $\tilde{H}=0$.

This algorithm ensures therefore the convergence of the system to the natural setpoint, even when the natural interval is unknown.

\section{Simulation Results}

The preceding linear stability analysis holds only for traffic conditions close to the nominal setpoint. In case of large deviations, nonlinear constraints have to be introduced mainly due to security requirements. In order to test the robustness of our linear approach under nonlinear conditions, we have developed a simulation software SIMETRO, which accounts for the nonlinearities and is based on a discrete-event approach where realistic security constraints are implemented. A full description of SIMETRO can be found in [12].

Our purpose in this section is to show through simulation results the economical performance of the proposed on-line traffic control. We consider a loop line with 30 platforms. One of these platforms is a terminus where the train staying time is adapted in order to ensure the periodicity of the nominal schedule. On the other hand, the train staying time at the terminus platform is also bounded below due to constraints on the line operation.

The traffic conditions are the following:

- the parameter $c=0.02$;

- the minimum staying time $S=15 \mathrm{~s}$ (except for the terminus platform);

- the nominal running times are between 60 and $100 \mathrm{~s}$;

- the minimal staying time at the terminus platform is 3 $\min$;

- the natural interval $H$ is $3 \mathrm{~min}$.

We consider three case studies.

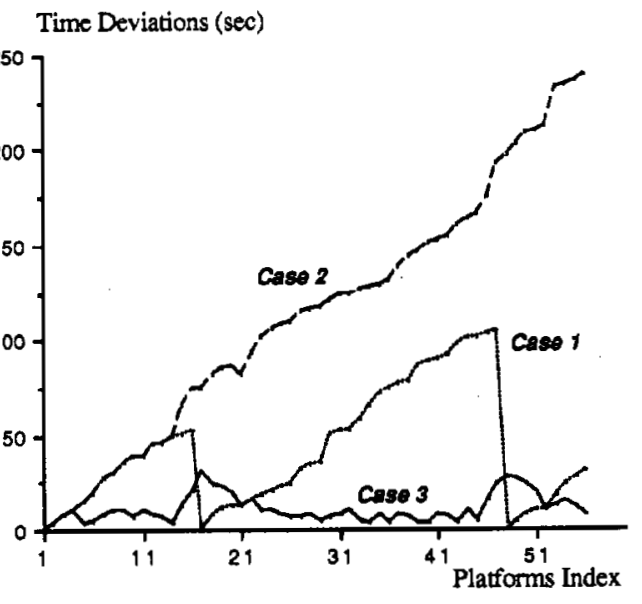

Fig. 5. Time deviations of train (1).

Case I:

- the total staying time at the terminus is 6 min constituted by the minimal time $(3 \mathrm{~min})$ and the time margin $(3 \mathrm{~min})$

- number of trains: 15

- the total nominal time for a full circuit is therefore $15^{*} 3$ $\min =45 \min$.

Case 2:

- the total staying time at the terminus is $3 \mathrm{~min}$

- no time margin

- number of trains: 14 .

The total nominal time for a full circuit is therefore $14 * 3$ $\min =42 \mathrm{~min}$. The reduction with respect to Case 1 is due to the suppression of the time margin at the terminus station. This allows us to operate the line with 14 trains instead of 15 .

Case 3:

- same conditions of operation as in Case 2 except that traffic control law (21) is implemented with $p=q=1$.

We simulate these three situations with the same sequence of stochastic disturbances applied to the system: the staying time at each train stop is disturbed with white noise having a mean of $3 \mathrm{~s}$ and standard deviation of $7 \mathrm{~s}$. In Fig. 5, we compare the evolution of the time deviations of train $\langle 1\rangle$ during its trip along the line. The platform numbers have to be interpreted modulo 30 .

Case 1: Due to the disturbances all the trains are delayed. The delay is increasing progressively along the circuit but the time margin is allowed to recover the nominal schedule at platform $\langle 1\rangle$

Case 2: Since there is no time margin the deviations are continuously increasing, from circuit to circuit.

Case 3: Due to the traffic regulation the delays are bounded (less than $40 \mathrm{~s}$ ) and are not increasing from circuit to circuit, even without a time margin at the terminus station.

These simulation results show the following.

1) The proposed traffic control law allows reduction in the terminus time margins and therefore the number of trains necessary to ensure a given nominal operation mode. This 
shows the economic benefit to be expected from efficient control.

2) The quality of the line operation (evaluated by the quality index $\Pi_{k}$ ) is better with traffic control than in Case 1 (exploitation with time margin at the terminus station).

\section{Conclusions}

In this paper, we have shown how efficient traffic control algorithms for high-frequency metro lines can be designed by means of a complete traffic model obtained from a discrete event approach.

1) We have developed a complete traffic analysis for sequential metro lines with or without reference to a nominal time schedule. The intrinsic instability of metro lines has been pointed out. We have shown how well chosen state-space formulations allow design of state-feedback control algorithms ensuring the stability of the system. The proposed implement in real-life systems.

2) Simulations have shown the robustness of the proposed traffic control algorithms against disturbances occurring randomly on a loop line and their efficiency when compared to a classical time margin strategy. Other simulation studies, including applications to the Brussels Metro network can be found, e.g., in [8], [9], [11], and [12].

\section{APPENDIX}

\section{A.1. Proof of Theorem 1}

Consider the matrix $H_{k}^{S}=\left(A_{k}^{S}\right)^{T} A_{k}^{S}$.

Its inverse $\left(H_{k}^{S}\right)^{-1}$ is given by traffic control algorithms have simple forms and are easy to

$$
\begin{aligned}
& \left|z-c_{k+1}^{2}-\left(1-c_{k+1}\right)^{2}\right| \leq 2 c_{k+1}\left(1-c_{k+1}\right) \\
& \left|z-c_{k+1}^{2}-\left(1-c_{k+1}\right)^{2}\right| \leq c_{k+1}\left(1-c_{k+1}\right) .
\end{aligned}
$$

Therefore, for any real $z$ inside the first circle (A1)

- if $z \geq\left(1-c_{k+1}\right)^{2} \Rightarrow 0<\left(1-c_{k+1}\right)^{2} \leq z \leq(1-$ $\left.c_{k+1}\right)<1$

- if $z<\left(1-c_{k+1}\right)^{2} \Rightarrow 0<\left(1-c_{k+1}\right)\left(1-2 \cdot c_{k+1}\right) \leq$ $z \leq\left(1-c_{k+1}\right)^{2}<1$.

For any real $z$ inside the second circle (A2)

- if $z \geq c_{k+1}^{2}+\left(1-c_{k+1}\right)^{2} \Rightarrow z \leq 1$

- if $z<c_{k+1}^{2}+\left(1-c_{k+1}\right)^{2} \Rightarrow 0<\left(1-2 c_{k+1}\right)^{2} \leq z$ $<c_{k+1}^{2}+\left(1-c_{k+1}\right)^{2}<1$.

For any real $z$ inside the third circle (A3)

- if $z \geq c_{k+1}^{2}+\left(1-c_{k+1}\right)^{2} \Rightarrow 1-2 c_{k+1}\left(1-c_{k+1}\right) \leq$ $z \leq 1-c_{k+1}\left(1-c_{k+1}\right)<1$

- if $z \geq c_{k+1}^{2}+\left(1-c_{k+1}\right)^{2} \Rightarrow 1-3 c_{k+1}\left(1-c_{k+1}\right) \leq$ $z \leq 1-2 c_{k+1}\left(1-c_{k+1}\right)<1$.

These three circles are therefore contained in the unit circle and the eigenvalues of $\left(H_{k}^{S}\right)^{-1}$ are, in absolute value, less than 1 (see 1)). This implies that the eigenvalues of $H_{k}^{S}$ are in absolute value strictly greater than 1 .

3) Let $\lambda_{\min }$ be the eigenvalue of $H_{k}^{S}$ with the smallest absolute value $\left(\left|\lambda_{\min }\right|>1\right)$. Then

$$
\begin{aligned}
\Gamma_{k+1} & =\frac{1}{I} \sum_{i=1}^{I}\left(x_{k+1}^{i}\right)^{2}=\frac{1}{I}\left\|X_{k+1}^{S}\right\|^{2}=\frac{1}{I}\left\|\left(X_{k}^{S}\right)^{T} H_{k}^{S} X_{k}^{S}\right\| \\
& \geq \frac{1}{I}\left|\lambda_{\min }\right|\left\|X_{k}^{S}\right\|^{2}>\frac{1}{I}\left\|X_{k}^{S}\right\|^{2}=\frac{1}{I} \sum_{i=1}^{I}\left(x_{k}^{i}\right)^{2}=\Gamma_{k} .
\end{aligned}
$$

$\left(H_{k}^{S}\right)^{-1}=\left[\begin{array}{ccccc}\left(1-c_{k+1}\right)^{2} & c_{k+1} \cdot\left(1-c_{k+1}\right) & & & \\ c_{k+1} \cdot\left(1-c_{k+1}\right) & c_{k+1}^{2}+\left(1-c_{k+1}\right)^{2} & c_{k+1} \cdot\left(1-c_{k+1}\right) & \ddots & 0 \\ 0 & \ddots & \ddots & \ddots & c_{k+1} \cdot\left(1-c_{k+1}\right) \\ & & & c_{k+1} \cdot\left(1-c_{k+1}\right) & c_{k+1}^{2}+\left(1-c_{k+1}\right)^{2}\end{array}\right]$.

Since $\left(H_{k}^{S}\right)^{-1}$ is symmetric, its eigenvalues are real.

1) No eigenvalue of $\left(H_{k}^{S}\right)^{-1}$ is equal to 1

$$
\begin{aligned}
\operatorname{det}\left[I_{I}-\left(H_{k}^{S}\right)^{-1}\right) & =c_{k+1}^{I}\left(1-c_{k+1}\right)^{I} \\
\cdot & {\left[I\left(2-c_{k+1}\right)-(I-1)\left(1-c_{k+1}\right)\right] \neq 0 }
\end{aligned}
$$

for $0<c_{k+1}<1$, where $I_{I}$ is the unity matrix of dimension I.

2) By the theorem of Gershgorin (see, e.g. [13]), we know that the eigenvalues of $\left(H_{k}^{S}\right)^{-1}$ are located in a set of three circles characterized by

$$
\left|z-\left(1-c_{k+1}\right)^{2}\right| \leq c_{k+1}\left(1-c_{k+1}\right)
$$

\section{A.2. Proof of Theorem 3}

1) Any realistic perturbation affecting the state vector is compatible with (16), i.e., it only affects the $M$ last components of this vector.

2) We first characterize the eigenvalues of $A_{L}$. Consider a first vector $U_{1}\left(\in R^{N}\right)$ of the form

$$
\begin{array}{r}
U_{1}=\left[\begin{array}{l}
V \\
0
\end{array}\right] \text { with } V=\left(v_{1}, v_{2}, \cdots, v_{N-M}\right)^{T} \\
\in R^{N-M}, V \neq 0 .
\end{array}
$$

Then obviously $\left\|A_{L} U_{1}\right\| \leq\left\|U_{1}\right\|$ and furthermore, if $v_{1} \neq$ $0,\left\|A_{L} U_{1}\right\|<\left\|U_{1}\right\|$. 
Consider a second vector $U_{2}\left(\in R^{N}\right)$ of the form

$U_{2}=\left[\begin{array}{l}0 \\ V\end{array}\right]$ with $V=\left(v_{1}, v_{2}, \cdots, v_{M}\right)^{T} \in R^{M}, \quad V \neq 0$

$$
\begin{aligned}
\left\|A_{L} U_{2}\right\|^{2} & =v_{1}^{2}+\left\|A_{22} \cdot V\right\|^{2} \geq\left\|A_{22} V\right\|^{2}>\|V\|^{2} \\
& =\left\|U_{2}\right\|^{2}
\end{aligned}
$$

where

$$
A_{22}=\left[\begin{array}{cc}
1 /(1-c) & -c /(1-c) \\
& 1 /(1-c) \\
0 &
\end{array}\right.
$$

Therefore $b=\left\|\lambda_{1}-a\right\|$ and $\lambda_{1}=1$. This means that

for $p>0$, all the eigenvalues are strictly inside the unit circle which implies that

$$
\begin{gathered}
\left\|Y_{j+1}^{i}\right\|<\left\|Y_{j}^{i}\right\|, \quad \Pi_{k+N}<\Pi_{k}, \quad \lim _{j \rightarrow \infty}\left\|Y_{j}^{i}\right\|=0 \text { and } \\
\lim _{1 \rightarrow \infty} \Pi_{k+1^{*} N}=0
\end{gathered}
$$

is an $(M \times M)$ matrix.

This shows that $A_{L}$ has at least one eigenvalue strictly inside and at least one eigenvalue strictly outside the unit circle.

3) Let $\nu_{0}$ denote the eigenvector corresponding to the eigenvalue $1\left(\nu_{0}=[1 \cdots 1]^{T}\right), \nu_{i}\left(i=1, n_{1}\right)$ the eigenvectors corresponding to the stable eigenvalues and $\eta_{i}\left(i=1, n_{2}\right)$ the eigenvectors corresponding to the unstable eigenvalues. Consider a state vector $X_{0}$ obtained by perturbing the equilibrium with a perturbation compatible with (16), $X_{0}$ is also expressed as

$X_{0}=\alpha_{0} \nu_{0}+\sum_{i=1}^{n_{1}} \alpha_{i} \nu_{i}+\sum_{j=1}^{n_{2}} \beta_{j} \eta_{j} \quad$ where $\quad \alpha_{i}, \beta_{j} \in R$.

As the perturbation is of the form of $U_{2}$ (see 2)), at least one of the coefficients $\beta_{j}$ is different from zero and the result follows immediately.

\section{A.3. Proof of Theorem 4}

The characteristic equation of $A_{L}(22)$ is

$$
\lambda^{N-M}(\lambda-a)^{M}-b^{M}=0
$$

where $a=(1-c) /\left[p+q+(1-c)^{2}\right]$ and $b=[q-c(1$ $-c)] /\left[p+q+(1-c)^{2}\right]$.

For $q>c(1-c)$, it can be easily checked that

1) for $p=0, a<1$, and $b=1-a$

2) for $p>0, a<1$ and $b<1-a$.

Assume that there exists $\lambda_{1}$, with $\left\|\lambda_{1}\right\| \geq 1$ satisfying the characteristic equation. Then

In this case

$$
1 \leq\left\|\lambda_{1}\right\|^{N-M}=\frac{b^{M}}{\left\|\lambda_{1}-a\right\|^{M}}\left({ }^{*}\right) .
$$

$$
\begin{gathered}
\text { for } \begin{aligned}
p=0, b^{2} & =(a-1)^{2} \leq\left\|a-\lambda_{2}\right\|^{2} \text { and } \\
\frac{b^{M}}{\left\|\lambda_{1}-a\right\|^{M}} & <1 \text {, which contradicts }\left(^{*}\right)
\end{aligned} \text { for } p=0, b^{2}=(a-1)^{2} \leq\left\|a-\lambda_{l}\right\|^{2} \text { and } \\
\frac{b^{M}}{\left\|\lambda_{1}-a\right\|^{M}} \leq 1 .
\end{gathered}
$$

for $p=0$, all the eigenvalues are strictly inside the unit circle except one, equal to 1 , with a corresponding eigenvector of the form $[1 \cdots 1]^{T}$. Without perturbation, the system converges therefore to a steady-state situation where all the deviations are equal and $\Pi_{k}$ is constant.

\section{A.4. Proof of Lemma 1}

In order to make the proof more comprehensible, we consider a simple loop line with 5 platforms and 3 trains. This proof can easily be extended to the general case at the price of tedious index manipulations. Consider a given step $j$, with $Y_{j}^{i}, \bar{U}_{j-1}^{j}$ and $\bar{W}_{j-1}^{i}$ defined as

$$
\begin{aligned}
& Y_{j}^{i}=\left[y_{1}^{3} y_{2}^{3} y_{3}^{3} y_{4}^{2} y_{5}^{1}\right]^{T} \quad \bar{U}_{j-1}^{i}=\left[u_{5}^{3} u_{1}^{3} u_{2}^{3} u_{3}^{2} u_{4}^{1}\right]^{T} \\
& \bar{W}_{j-1}^{i}=\left[w_{5}^{3} w_{1}^{3} w_{2}^{3} w_{3}^{2} w_{4}^{1}\right]^{T} .
\end{aligned}
$$

Obviously, these vectors are defined in relation with train 3 . The components of $Y_{j}^{i}$ are related to the departure times $t_{k}$ by

$$
\begin{gathered}
y_{1}^{3}=t_{1}^{3}-t_{1}^{2}-H, \quad y_{2}^{3}=t_{2}^{3}-t_{2}^{2}-H, \\
y_{3}^{3}=t_{3}^{3}-t_{3}^{2}-H, \quad y_{4}^{2}=t_{4}^{2}-t_{4}^{1}-H, \\
y_{5}^{1}=t_{5}^{1}-t_{5}^{3}-H\left(^{*}\right) .
\end{gathered}
$$

On the other hand, these 10 values $t_{k}^{i}$ satisfy a set of 5 transfer equations (4) denoted $\left({ }^{* *}\right)$

$c\left(t_{1}^{3}-t_{1}^{2}-H\right)=-c H+t_{1}^{3}-t_{5}^{3}-S-R_{5}-u_{5}^{3}-w_{5}^{3}$ $c\left(t_{2}^{3}-t_{2}^{2}-H\right)=-c H+t_{2}^{3}-t_{1}^{3}-S-R_{1}-u_{1}^{3}-w_{1}^{3}$ $c\left(t_{3}^{3}-t_{3}^{2}-H\right)=-c H+t_{3}^{3}-t_{2}^{3}-S-R_{2}-u_{2}^{3}-w_{2}^{3}$ $c\left(t_{4}^{2}-t_{4}^{1}-H\right)=-c H+t_{4}^{2}-t_{3}^{2}-S-R_{3}-u_{3}^{2}-w_{3}^{2}$ $c\left(t_{5}^{1}-t_{5}^{3}-H\right)=-c H+t_{5}^{1}-t_{4}^{1}-S-R_{4}-u_{4}^{1}-w_{4}^{1}$

The relations $(*)$ and $\left({ }^{* *}\right)$ constitute a set of 10 linear relations between these $10 t_{k}^{i}$ and the vectors $\bar{U}_{j-1}^{i}$ and $\bar{W}_{j-1}^{i}$. The vectors $Y_{j}^{i}, \bar{U}_{j-1}^{i}$ and $\bar{W}_{j-1}^{i}$ are compatible with the structure of the line if there exists a solution, in the unknown $t_{k}^{i}$, for this linear nonhomogeneous equations system. Obviously, this solution is not unique: the $t_{k}^{i}$ are defined 
modulo an additive constant. This means that the $(10 \times 10)$ matrix of the coefficients $t_{k}^{i}$ is not full-rank (it can be checked that the rank is 9). There exists therefore a compatibility condition between $Y_{j}^{i}, \bar{U}_{j-1}^{i}$, and $\bar{W}_{j-1}^{i}, R_{i}(i=$ $1, \cdots, 5), H$ and $S$. This condition is obtained by summing the relations $\left(^{* *}\right)$. Using $\left({ }^{*}\right)$, we obtain

$$
\begin{array}{r}
{\left[\begin{array}{lllll}
c & c & c-1 & c-1 & c-1
\end{array}\right] Y_{j}^{i}+\left[\begin{array}{lllll}
1 & 1 & 1 & 1 & 1
\end{array}\right]} \\
\cdot\left(\bar{U}_{j-1}^{l}+\bar{W}_{j-1}^{i}\right)=-\left[\sum_{k=1}^{5} R_{k}+5 S+5 c H\right]=0
\end{array}
$$

or

$$
b_{1}^{T} Y_{j}^{i}+b_{2}^{T}\left[\bar{U}_{j-1}^{i}+\bar{W}_{j-1}^{i}\right]=0 .
$$

\section{A.5. Proof of Theorem 6}

The control law (31) coincides with the control law (21), with $p=0$ provided that a reference time schedule compatible with the natural interval $H$ is used. Let us therefore analyze the closed-loop behavior in the time deviations $\left(X_{j}^{i}\right)$ formulation.

For $p=0$ and $q>c(1-c), \bar{A}_{L}$ has one eigenvalue equal to 1 with a corresponding eigenvector $v_{1}=[1 \cdots 1]^{T}$ and the other eigenvalues strictly inside the unit circle (see Theorem 4). Let $\left(\lambda_{i}, v_{i}\right)(1 \leq i \leq N)$ denote the couples (eigenvalues, eigenvectors). There exist $N$ constant $\alpha_{i}(i=$ $1, \cdots, N)$ such that the initial condition $X_{0}^{h}(h \in\{1, \cdots, M\}$ can be expressed in terms of $v_{i}(i=1, \cdots, N)$

$$
X_{0}^{h}=\sum_{j=1}^{N} \alpha_{i} v_{i}
$$

Therefore, for the disturbance free system

$$
X_{j}^{h}=\alpha_{1} v_{1}+\sum_{i=2}^{N} \alpha_{i}\left(\lambda_{i}\right)^{j} v_{i}
$$

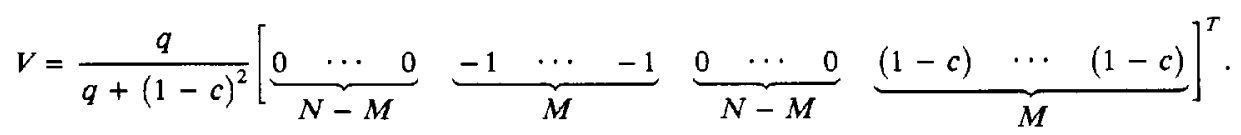

It follows that $\left\|X_{j+1}^{h}\right\| \leq\left\|X_{j}^{h}\right\|$ and $\lim _{j \rightarrow \infty} X_{j}^{h}=\alpha_{1} v_{1}$ and it can be easily checked that

$$
\begin{gathered}
\left\|Y_{j+1}^{h}\right\| \leq\left\|Y_{j}^{h}\right\|, \quad \lim _{j \rightarrow \infty} Y_{j}^{h}=0, \quad \sigma_{k+1^{*} N} \leq \sigma_{k}, \\
\lim _{1 \rightarrow \infty} \sigma_{k+1^{*} N}=0 \text { and } \lim _{j \rightarrow \infty}\left\|\bar{U}_{j}^{h}\right\|=0 .
\end{gathered}
$$

\section{A.6. Proof of Lemma 2}

1) It can easily be checked that 1 is an eigenvalue of the closed-loop matrix (32) with a corresponding eigenvector of the given form. This vector is not orthogonal to $b$.

2) Let us denote $M$ the closed-loop matrix (32). It can be checked that $M^{T} b=b$. Therefore, the eigenvectors corresponding to the eigenvalues not equal to 1 are orthogonal to $b$.

From Theorem 6, we conclude that the eigenvalues corresponding to the $(2 N-1)$ eigenvectors orthogonal to $b$ are strictly inside the unit circle.

\section{A.7. Proof of Theorem 7}

Let $\lambda_{i}$ and $v_{i}(i=1, \cdots, 2 N)$ denote the eigenvalues of the closed-loop matrix defined in (32). The initial condition as well as the input vector can be expressed in terms of the eigenvectors $v_{i}$.

$$
\begin{array}{r}
{\left[\begin{array}{c}
Y_{1}^{h} \\
\bar{U}_{0}^{h}
\end{array}\right]=\sum_{i=1}^{2 N} \alpha_{i} v_{i} \text { and }\left[\begin{array}{c}
B_{L} \\
0_{N-M, M} \\
I_{M}
\end{array}\right] \Delta W_{j}^{h}=\sum_{i=1}^{2 N} \beta_{i}(j) v_{i}} \\
(h \in\{1, \cdots, M\}) .
\end{array}
$$

Furthermore, as $\bar{W}_{j}^{h}$ is bounded, $\sum_{j=1}^{1} \beta_{i}(j)$ is bounded for each $i(i=1, \cdots, 2 N)$ and $j$. The state vector can be expressed as

$$
\left[\begin{array}{c}
Y_{j}^{h} \\
\bar{U}_{j-1}^{h}
\end{array}\right]=\sum_{i=1}^{2 N} \alpha_{i}\left(\lambda_{i}\right)^{j-1} v_{i}+\sum_{k=1}^{j-1}\left[\sum_{i=1}^{2 N} \beta_{i}(k)\left(\lambda_{i}\right)^{j-k} v_{i}\right]
$$

and its norm is bounded by

$$
\sum_{i=1}^{2 N}\left|\alpha_{i}\right|\left\|v_{i}\right\|+\sum_{i=1}^{2 N}\left|\left[\sum_{k=1}^{j-1} \beta_{i}(k)\right]\right|\left\|u_{i}\right\|
$$

which is bounded.

\section{A.8. Proof of Theorem 8}

In absence of disturbances, the system satisfies

$\left[\begin{array}{c}Y_{j+1}^{h} \\ U_{j}^{h}\end{array}\right]=\left[\begin{array}{cc}A_{L}+B_{L} K_{1} & B_{L} K_{2} \\ 0_{N-M, M} & S_{N}+0_{N-M, M} \\ K_{1} & K_{2}\end{array}\right]\left[\begin{array}{c}Y_{j}^{h} \\ U_{j-1}^{h}\end{array}\right]+\tilde{H} V$

where $h \in\{1, \cdots, M\}$ and $V$ is the $2 N$-vector defined by

From Theorem 6 and Lemma 2, we know that, for any initial condition compatible with the line structure, the state of this system converges to a stable equilibrium point. It is easy to check that this point corresponds to steady-state components of $Y$ and $U$, say $y^{*}$ and $u^{*}$, such that

$$
q y^{*}+(1-c) u^{*}=-q \tilde{H} .
$$

On the other hand, this steady-state point is compatible with the line structure. Therefore

$$
(N c-M) y^{*}+K u^{*}=0 \text {. }
$$

The relations (A8.1), (A8.2), and (A8.3) determine $y^{*}$ and $u^{*}$. The steady-state setpoint is characterized by $u^{*}$ and $H^{*}=H+\mathrm{y}^{*}$, and the result follows immediately.

\section{ACKNOWLEDGMENT}

Prof. M. Polis, Associate Editor at Large, is gratefully acknowledged for helpful suggestions for improving the read- 
ability of the paper. This paper presents research results of the Belgian program on concerted research actions and on interuniversity attraction poles initiated by the Belgian State Prime Minister's Office-Science Policy Programming. The scientific responsibility rests with its authors.

\section{REFERENCES}

[1] W. S. Levine and M. Athans, "On the optimal error regulation of string of moving vehicles," IEEE Trans. Automat. Contr., vol. AC-11, pp. 355-361, 1966.

[2] S. Meizer and B. C. Kuo "A closed form solution for the optimal error regulation of a string of moving vehicles." IEEE Trans. Automat. Contr., vol. AC-16, pp. 50-52, Feb. 1971.

[3] L. E. Peppard and V. Gourishankar, "Optimal control of a string of moving vehicles," IEEE Trans. Automat. Contr., vol. AC-15, pp. 386-387, June 1970 .

[4] K. C. Chu, "Optimal decentralized regulation of a string of coupled systems," IEEE Trans. Automat. Contr, vol. AC-19, pp. 243-246, June 1974.

[5] T. Yoshikawa, H. Oka, and H. Hanafusa, "Decentralized control of moving vehicles on a loop line," in Proc. IFAC Conf. Large Scale Syst., Warsaw, Poland, 1983, pp. 183-188.

[6] J. E. Cury, F. A. Gomide, and M. J. Mendes, "A methodology for generation of optimal schedules for an underground railway system," IEEE Trans. Automat. Contr., vol. AC-25, no. 2, pp. 217-222, Apr. 1980.

[7] S. Araya and S. Sone, "Traffic dynamics of automated transit systems with pre-established schedules," IEEE Trans. Syst., Man Cybern., vol. 14, pp. 677-687, July 1984

[8] G. Campion, V. Van Breusegem, P. Pinson, and G. Bastin, "Traffic regulation of an underground railway transportation system by state feedback," Optimal Contr. Appl. Methods, vol. 6, pp. 385-402, Dec. 1985.

[9] V. Van Breusegem, "Simulation et régulation de lignes de métro automatisées," Ph.D. dissertation, Univ. Louvain, Louvain, Belgium, Oct. 1987

[10] G. Campion, V. Van Breusegem, and G. Bastin, "Modeling, simulation and control of the traffic at the terminus stations of urban underground railway lines," in Proc. 5th IFAC Symp. Cont. Transportation Syst., Vienna, Austria, July 1986, pp. 259-264.

[11] V. Van Breusegem, G. Campion, and G. Bastin, "State feedback traffic control of the transient behaviour of underground public transportation systems," in Proc. 10th IFAC World Congress Automat. Contr., Munich, Germany, Aug. 1987, pp. 257-262.

[12] - "Simulation and control of the traffic of metro lines," in Proc. European Simulation Multiconference, Conf. Simulation Traffic Contr., Nice, France, June 1988, pp. 169-175.

[13] B. C. Kuo, Digital Control Systems. Tokyo, Japan: Holt Saunders Internat. Editions, 1980.

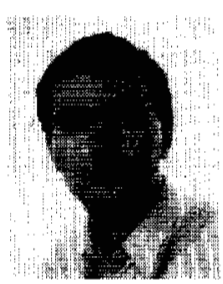

Vincent Van Breusegem was born in Charleroi, Belgium, in 1960 . He received the electrical engineering degree and the Ph.D. degree, both from Université Catholique de Louvain, Louvain-laNeuve, Belgium, in 1984 and 1987 , respectively.

Since 1984 , he has been with the Laboratoire d'Automatique, Dynamique et Analyse des Systèmes as a Research Assistant excepted in 1990 when he spent a year at the Laboratoire d'Automatique de Grenoble, France. His research interests are in discrete-event systems, transportation systems, and biotechnological processes.

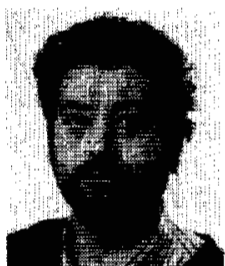

Guy Campion received the mechanical engineering degree and the Ph.D. degree, both from Université Catholique de Louvain, Louvain-la-Neuve, Belgium.

Since 1977, he has been a Research Associate (Fonds National de la Recherche Scientifique), with the Laboratoire d'Automatique, Dynamique et Analyse des Systèmes. His main research interests are in nonlinear control theory (with applications in mechanical systerns) and transportation systems.

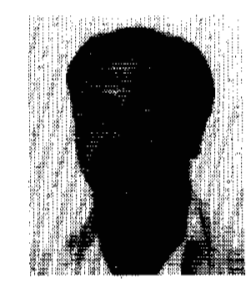

Georges Bastin received the electrical engineering degree and the Ph.D. degree, both from Université Catholique de Louvain, Louvain-la-Neuve, Belgium.

Since 1975, he has been with the Laboratoire d'Automatique, Dynamique et Analyse des Systèmes where he has been the head of the laboratory from 1983 to 1989 . His main research interests are in system identification, nonlinear control theory, adaptive systems, and random fields with applications to mechanical systems, biological processes, transportation systems and hydrology. 